\title{
Two applications of the spectrum of numbers
}

\author{
Christiane Frougny*and Edita Pelantová ${ }^{\dagger}$
}

\begin{abstract}
Let the base $\beta$ be a complex number, $|\beta|>1$, and let $A \subset \mathbb{C}$ be a finite alphabet of digits. The $A$-spectrum of $\beta$ is the set $S_{A}(\beta)=\left\{\sum_{k=0}^{n} a_{k} \beta^{k} \mid n \in\right.$ $\left.\mathbb{N}, a_{k} \in A\right\}$. We show that the spectrum $S_{A}(\beta)$ has an accumulation point if and only if 0 has a particular $(\beta, A)$-representation, said to be rigid.

The first application is restricted to the case that $\beta>1$ and the alphabet is $A=\{-M, \ldots, M\}, M \geq 1$ integer. We show that the set $Z_{\beta, M}$ of infinite $(\beta, A)$-representations of 0 is recognizable by a finite Büchi automaton if and only if the spectrum $S_{A}(\beta)$ has no accumulation point. Using a result of Akiyama-Komornik and Feng, this implies that $Z_{\beta, M}$ is recognizable by a finite Büchi automaton for any positive integer $M \geq\lceil\beta\rceil-1$ if and only if $\beta$ is a Pisot number. This improves the previous bound $M \geq\lceil\beta\rceil$.

For the second application the base and the digits are complex. We consider the on-line algorithm for division of Trivedi and Ercegovac generalized to a complex numeration system. In on-line arithmetic the operands and results are processed in a digit serial manner, starting with the most significant digit. The divisor must be far from 0 , which means that no prefix of the $(\beta, A)$ representation of the divisor can be small. The numeration system $(\beta, A)$ is said to allow preprocessing if there exists a finite list of transformations on the divisor which achieve this task. We show that $(\beta, A)$ allows preprocessing if and only if the spectrum $S_{A}(\beta)$ has no accumulation point.
\end{abstract}

Key words: spectrum, Pisot number, Büchi automaton

Mathematics Subject Classification: 11K16, 68Q45

\section{Introduction}

The so-called beta-numeration has been introduced by Rényi in 21 and studied by Parry in 20] in the case that $\beta$ is a real number, $\beta>1$, and since then there are been many works in this domain, in connection with number theory, dynamical systems, and automata theory, see the survey [12] or more recent [22] for instance.

For $\beta>1$ and $M \geq 1$ an integer, the following spectrum

$$
X_{M}(\beta)=\left\{\sum_{k=0}^{n} a_{k} \beta^{k} \mid n \in \mathbb{N}, a_{k} \in\{0,1, \ldots, M\}\right\}
$$

has been introduced by Erdős, Joó and Komornik 8 .

Since $X_{M}(\beta)$ is discrete its elements can be arranged into an increasing sequence

$$
0=x_{0}<x_{1}<\cdots
$$

Denote $\ell_{M}(\beta)=\liminf _{k \rightarrow \infty}\left(x_{k+1}-x_{k}\right)$. Numerous works have been devoted to the study of this value, see in particular the introduction and the results of [1].

\footnotetext{
*IRIF, UMR 8243 CNRS and Université Paris-Diderot

${ }^{\dagger}$ Doppler Institute for Mathematical Physics and Applied Mathematics, and Department of Mathematics, FNSPE, Czech Technical University in Prague
} 
More generally, let $\beta$ be a complex number, $|\beta|>1$, and let $A \subset \mathbb{C}$ be a finite alphabet of digits. The $A$-spectrum of $\beta$ is the set

$$
S_{A}(\beta)=\left\{\sum_{k=0}^{n} a_{k} \beta^{k} \mid n \in \mathbb{N}, a_{k} \in A\right\} .
$$

Recently Feng answered an open question raised in [8], see also [1, on the density of the spectrum of $\beta$ when $\beta$ is real and the digits are consecutive integers:

Theorem 1.1 ([9]). Let $\beta>1$ and let $A=\{-M, \ldots, M\}, M$ an integer $\geq 1$. Then the spectrum $S_{A}(\beta)$ is dense in $\mathbb{R}$ if and only if $\beta<M+1$ and $\beta$ is not a Pisot number.

Feng has obtained the following corollary: $\ell_{M}(\beta)=0$ if and only if $\beta<M+1$ and $\beta$ is not a Pisot number.

In this paper we use the concept of spectrum of a number to solve two problems arising in beta-numeration.

Let $\beta$ and the digits of $A$ be complex. The topological properties of the spectrum are linked with a particular representation of 0 . Let $z_{1} z_{2} \cdots$ be a $(\beta, A)$ representation of 0 , that is to say, $\sum_{i>1} z_{i} \beta^{-i}=0$. It is said to be rigid if $0 \cdot z_{1} z_{2} \cdots z_{j} \neq 0.0 z_{2}^{\prime} \cdots z_{j}^{\prime}$ for all $j \geq 2$ and for all $z_{2}^{\prime} \cdots z_{j}^{\prime}$ in $A^{*}$. The term "rigid" comes from the preprocessing motivation, see Section 5 .

We first prove that the spectrum $S_{A}(\beta)$ has an accumulation point if and only if 0 has a rigid $(\beta, A)$-representation, Theorem 3.5 .

Then we obtain some results when the base is a complex Pisot number, which extend the real case covered by Garsia [13]. Let $\beta$ be a complex number, and let $A \subset \mathbb{Q}(\beta)$ containing 0 . If $\beta$ is real and if $\beta$ or $-\beta$ is a Pisot number, or if $\beta \in \mathbb{C} \backslash \mathbb{R}$ is a complex Pisot number then $S_{A}(\beta)$ has no accumulation point, Theorem 3.6.

The first question we address in this work is the one of the recognizability by a finite Büchi automaton of the set of infinite $\beta$-representations of 0 when $\beta$ is a real number and the digits are integer.

The set of infinite $\beta$-representations of 0 on the alphabet $\{-M, \ldots, M\}, M \geq 1$ integer, is denoted

$$
Z_{\beta, M}=\left\{z_{1} z_{2} \cdots \mid \sum_{i \geq 1} z_{i} \beta^{-i}=0, z_{i} \in\{-M, \ldots, M\}\right\} .
$$

The following result has been formulated in [12]:

Theorem 1.2. Let $\beta>1$. The following conditions are equivalent:

1. the set $Z_{\beta, M}$ is recognizable by a finite Büchi automaton for every integer $M$,

2. the set $Z_{\beta, M}$ is recognizable by a finite Büchi automaton for one integer $M \geq$ $\lceil\beta\rceil$,

\section{3. $\beta$ is a Pisot number.}

(3) implies (1) is proved in [10, (1) implies (3) is proved in [2] and (2) implies (1) is proved in [11.

Note that in [7] Bugeaud has shown, using (1) implies (3) of Theorem 1.2, that if $\beta$ is not a Pisot number then there exists an integer $M$ such that $\ell_{M}(\beta)=0$.

In this paper we first prove that the set $Z_{\beta, M}$ is recognizable by a finite Büchi automaton if and only if the spectrum $S_{A}(\beta)$ has no accumulation point, Theorem 4.2 . 
By [1] or [9] it is known that, for $A=\{-M, \ldots, M\}$, the spectrum $S_{A}(\beta)$ has an accumulation point if and only if $\beta<M+1$ and $\beta$ is not Pisot.

This result together with Theorem 4.2 proves the conjecture stated in [12]:

If the set $Z_{\beta,[\beta]-1}$ is recognizable by a finite Büchi automaton then $\beta$ must be a Pisot number.

Moreover we obtain a simpler proof of the implication $(2) \Rightarrow(3)$ of Theorem 1.2 . Note that the value $M=\lceil\beta\rceil-1$ is the best possible as $Z_{\beta, M}$ is reduced to $\left\{0^{\omega}\right\}$ if $M<\lceil\beta\rceil-1$.

Normalization in base $\beta$ is the function which maps any $\beta$-representation on the canonical alphabet $A_{\beta}=\{0, \ldots,\lceil\beta\rceil-1\}$ of a number $x \in[0,1]$ onto the greedy $\beta$-expansion of $x$. Since the set of greedy $\beta$-expansions of the elements of $[0,1]$ is computable by a finite Büchi automaton when $\beta$ is a Pisot number, see [4], the following result holds true:

Normalization in base $\beta>1$ is computable by a finite Büchi automaton on the alphabet $A_{\beta} \times A_{\beta}$ if and only if $\beta$ is a Pisot number.

The second utilisation of the notion of spectrum occurs in the on-line algorithm for division in a complex base.

On-line arithmetic, introduced in [25] for an integer base, is a mode of computation where operands and results are processed in a digit serial manner, starting with the most significant digit. To generate the first digit of the result, the first $\delta$ digits of the operands are required. The integer $\delta$ is called the delay of the algorithm. One of the interests of the functions that are on-line computable is that they are continuous for the usual topology on the set of infinite words on a finite alphabet.

In [5, 6] we have extended the original on-line algorithm of Trivedi-Ercegovac to a complex base. The algorithm for on-line division in a complex numeration system $(\beta, A)$ has two parameters: the delay $\delta \in \mathbb{N}$ and $D>0$, the minimal value (in modulus) of the divisor.

When making division, we need that the divisor stays away from 0 . By definition of the on-line algorithm, this means that the value of all the prefixes of the divisor $d_{1} d_{2} \cdots$ must be greater in absolute value than $D>0$, so the divisor must be preprocessed before making the division.

We say that a complex numeration system $(\beta, A)$ allows preprocessing if there exists a finite list of transformations on the $(\beta, A)$-representation of the divisor which achieve this task, see Definition 5.1

We show that a complex numeration system $(\beta, A)$ allows preprocessing if and only if the spectrum $S_{A}(\beta)$ has no accumulation point, Theorem 5.4.

\section{Preliminaries}

\subsection{Words and automata}

Let $A$ be a finite alphabet. A finite word $w$ on $A$ is a finite concatenation of letters from $A, w=w_{1} \cdots w_{n}$ with $w_{i}$ in $A$. The set of all finite words over $A$ is denoted by $A^{*}$. An infinite word $w$ on $A$ is an infinite concatenation of letters from $A$, $w=w_{1} w_{2} \cdots$ with $w_{i}$ in $A$. The set of all infinite words over $A$ is denoted by $A^{\mathbb{N}}$. The infinite concatenation $u u u \cdots$ is noted $u^{\omega}$. If $w=u v, u$ is a prefix of $w$.

An automaton $\mathcal{A}=(A, Q, I, T)$ over the alphabet $A$ is a directed graph labeled by letters of $A$, with a denumerable set $Q$ of vertices called states. $I \subseteq Q$ is the set of initial states, and $T \subseteq Q$ is the set of terminal states. The automaton is said to be finite if the set of states $Q$ is finite.

An infinite path of $\mathcal{A}$ is said to be successful if it starts in $I$ and goes infinitely often through $T$. The set of infinite words recognized by $\mathcal{A}$ is the set of labels of 
its successful infinite paths. An automaton used to recognize infinite words in this sense is called a Büchi automaton.

\subsection{Numeration}

Let $\beta$ be a complex number, $|\beta|>1$, and let $A \subset \mathbb{C}$ be a finite set, the alphabet of digits. We say that $(\beta, A)$ is a numeration system. A $(\beta, A)$-representation of a number $z$ is an infinite word $z_{1} z_{2} \cdots$ such that $z=\sum_{i=1}^{+\infty} z_{i} \beta^{-i}$ with $z_{i}$ in $A$. It should be noted that here we do not make any hypothesis on the fact that every complex number has, or does not have, a $(\beta, A)$-representation. This is a difficult problem, studied by many authors, see the pioneering works of Knuth [17, Kátai and Kovács [16], Gilbert [14, Thurston [24] for instance.

We now recall some definitions and results on the so-called beta-numeration, see [12] or [22] for a survey. Let $\beta>1$ be a real number. Any real number $x \in[0,1]$ can be represented by a greedy algorithm as $x=\sum_{i=1}^{+\infty} x_{i} \beta^{-i}$ with $x_{i}$ in the canonical alphabet $A_{\beta}=\{0, \ldots,\lceil\beta\rceil-1\}$ for all $i \geq 1$. The greedy sequence $\left(x_{i}\right)_{i \geq 1}$ corresponding to a given real number $x$ is the greatest in the lexicographical order, and is said to be the $\beta$-expansion of $x$, see 21]. It is denoted by $d_{\beta}(x)=\left(x_{i}\right)_{i \geq 1}$. When the expansion ends in infinitely many 0 's, it is said to be finite, and the 0's are omitted.

The greedy $\beta$-expansion of 1 is denoted $d_{\beta}(1)=\left(t_{i}\right)_{i \geq 1}$. When it is finite, of the form $d_{\beta}(1)=t_{1} \cdots t_{m}$, the quasi-greedy $\beta$-expansion of 1 is defined as $d_{\beta}^{*}(1)=$ $\left(t_{1} \cdots t_{m-1}\left(t_{m}-1\right)\right)^{\omega}$. If it is infinite, set $d_{\beta}^{*}(1)=d_{\beta}(1)$. The sequence $d_{\beta}^{*}(1)$ is the lexicographically greatest infinite representation of 1 in the base $\beta$ and the alphabet $\mathbb{N}$. It is known from [20] that a sequence of integers $x_{1} x_{2} \cdots$ is the greedy $\beta$-expansion of some $x$ from $[0,1]$ if and only if, for all $j \geq 1, x_{j} x_{j+1} \cdots$ is less than or equal to $d_{\beta}^{*}(1)$ in the lexicographic order.

Notation: The numerical value $y_{m-1} \beta^{m-1}+\cdots+y_{0}+y_{-1} \beta^{-1}+y_{-2} \beta^{-2}+\cdots$ is denoted by $y_{m-1} \cdots y_{0} \cdot y_{-1} y_{-2} \cdots$.

\section{$2.3 \quad$ Numbers}

A number $\beta>1$ such that $d_{\beta}(1)$ is eventually periodic is a Parry number. It is a simple Parry number if $d_{\beta}(1)$ is finite.

A Pisot number is an algebraic integer greater than 1 such that all its Galois conjugates have modulus less than 1. Every Pisot number is a Parry number, see 3 and 23 .

A complex Pisot number is an algebraic integer $\beta$ such that $|\beta|>1$ and such that all its Galois conjugates different from its complex conjugate $\bar{\beta}$ have modulus less than 1 .

\section{Spectrum and rigid representation of 0}

Let $\beta$ be a complex number, $|\beta|>1$, and let $A \subset \mathbb{C}$ be a finite alphabet. We introduce the $A$-spectrum of $\beta$ as

$$
S_{A}(\beta)=\left\{\sum_{k=0}^{n} a_{k} \beta^{k} \mid n \in \mathbb{N}, a_{k} \in A\right\} .
$$

The topological properties of $S_{A}(\beta)$ are linked with a particular representation of 0 . 
Definition 3.1. Let $z_{1} z_{2} \cdots$ be a $\beta$-representation of 0 on $A$, that is to say, $\sum_{i \geq 1} z_{i} \beta^{-i}=0$. It is said to be rigid if $0 \cdot z_{1} z_{2} \cdots z_{j} \neq 0.0 z_{2}^{\prime} \cdots z_{j}^{\prime}$ for all $j \geq 2$ and for all $z_{2}^{\prime} \cdots z_{j}^{\prime}$ in $A^{*}$.

Example 3.2. The signed digit $(-1)$ is denoted $\overline{1}$. In base 2 with alphabet $\{\overline{1}, 0,1\}$, 0 has two representations, namely $0=0.1 \overline{1} \overline{1} \overline{1} \cdots=0 . \overline{1} 1111 \cdots$. They are not rigid, since $0.1 \overline{1}=0.01$ and $0 . \overline{1} 1=0.0 \overline{1}$.

Definition 3.3. Let $z_{1} z_{2} \cdots$ be a $(\beta, A)$-representation of 0 . For $n$ in $\mathbb{N}$, its $n$-th tail is $r_{n}=0 \cdot z_{n+1} z_{n+2} z_{n+3} \cdots$.

Lemma 3.4. Let $z_{1} z_{2} \cdots$ be a $(\beta, A)$-representation of 0 .

1. If the sequence $\left(r_{n}\right)_{n \in \mathbb{N}}$ is injective, then the spectrum $S_{A}(\beta)$ has an accumulation point.

2. If the representation of 0 is rigid, then the sequence $\left(r_{n}\right)_{n \in \mathbb{N}}$ is injective.

Proof. Since $0=0 \cdot z_{1} z_{2} z_{3} \cdots$, the $n^{\text {th }}$ tail $r_{n}=\sum_{k=1}^{+\infty} z_{n+k} \beta^{-k}=-\sum_{k=0}^{n-1} z_{n-k} \beta^{k}$. It means that $-r_{n}$ belongs to the spectrum $S_{A}(\beta)$ and moreover

$$
\left|r_{n}\right| \leq \frac{\alpha}{|\beta|-1}, \quad \text { where } \alpha=\max \{|a|: a \in A\} .
$$

1) If the sequence $\left(r_{n}\right)_{n \in \mathbb{N}}$ is injective, then the ball centered at 0 with radius $\frac{\alpha}{|\beta|-1}$ contains infinitely many elements $\left(-r_{n}\right)$ of the spectrum, and thus the spectrum has an accumulation point.

2) Suppose that the representation of 0 is rigid. We show by contradiction the injectivity of $\left(r_{n}\right)_{n \in \mathbb{N}}$. Let us assume that $r_{i}=r_{j}$ for some indices $i<j$. Then $\sum_{k=0}^{j-1} z_{j-k} \beta^{k}=\sum_{k=0}^{i-1} z_{i-k} \beta^{k}$ and thus $0 \cdot z_{1} z_{2} \cdots z_{j}=0 \cdot \underbrace{0 \cdots 0}_{(j-i) \text { times }} z_{1} \cdots z_{i}-$ a contradiction with the rigidity of the representation of zero.

Theorem 3.5. Let $\beta$ be a complex number, $|\beta|>1$, and let $A \subset \mathbb{C}$ be a finite alphabet. The spectrum $S_{A}(\beta)$ has an accumulation point if and only if 0 has a rigid $(\beta, A)$-representation.

Proof. $(\Rightarrow)$ Let $s$ be an accumulation point of $S_{A}(\beta)$. There exists an injective sequence $\left(x^{(n)}\right)_{n \in \mathbb{N}}$ of points from $S_{A}(\beta)$ such that $\lim \left(x^{(n)}\right)_{n \in \mathbb{N}}=s$. For any $x \in S_{A}(\beta)$ denote

$$
\rho(x)=\min \left\{n \in \mathbb{N}: x=\sum_{k=0}^{n} a_{k} \beta^{k}, \text { with } a_{k} \in A\right\} .
$$

Set $\rho_{n}=\rho\left(x^{(n)}\right)$, then $x^{(n)}=\sum_{k=0}^{\rho_{n}} x_{k}^{(n)} \beta^{k}$. The sequence $\left(\rho_{n}\right)_{n \in \mathbb{N}}$ is unbounded, as there exists only a finite number of strings of a given length over a finite alphabet. Without loss of generality assume that $\left(\rho_{n}\right)_{n \in \mathbb{N}}$ is strictly increasing. Clearly,

$$
\frac{x^{(n)}}{\beta^{1+\rho_{n}}}=0 \cdot x_{\rho_{n}}^{(n)} \cdots x_{2}^{(n)} x_{1}^{(n)} x_{0}^{(n)} 0000 \cdots \rightarrow 0
$$

since the nominators tend to $s$. The fact that $A^{\mathbb{N}}$ endowed with the product topology is a compact space implies the existence of a string $x_{1} x_{2} x_{3} \cdots$ which is the limit of a subsequence of $\left(x_{\rho_{n}}^{(n)} \cdots x_{2}^{(n)} x_{1}^{(n)} x_{0}^{(n)} 0^{\omega}\right)_{n \in \mathbb{N}}$. It means that for any $N \in \mathbb{N}$ one can find $n \in \mathbb{N}$ such that $\rho_{n}>N$ and $x_{\rho_{n}}^{(n)} \cdots x_{2}^{(n)} x_{1}^{(n)} x_{0}^{(n)}$ is a prefix of $x_{1} x_{2} x_{3} \cdots$. The 
definition of $\rho_{n}$ and the fact (3.1) forces $0 . x_{1} x_{2} x_{3} \cdots$ to be a rigid representation of 0 .

$(\Leftarrow) \quad$ Let $0=0 \cdot z_{1} z_{2} z_{3} \cdots$ be a rigid representation of zero. Then by Point 2 of Lemma 3.4 the sequence of its tails is injective and by Point 1 of the same lemma, the spectrum has an accumulation point.

We now turn to the Pisot case. The real case is due to Garsia [13, and we follow his idea.

Theorem 3.6. Let $\beta$ be a complex number, $|\beta|>1$, and let $A \subset \mathbb{Q}(\beta)$ containing 0 .

1. If $\beta$ is real and if $\beta$ or $-\beta$ is Pisot

2. or if $\beta \in \mathbb{C} \backslash \mathbb{R}$ is complex Pisot

then $S_{A}(\beta)$ has no accumulation point.

Proof. Let $\beta=\beta_{1}$ be a complex Pisot number of degree $r$ with conjugates $\beta_{2}=$ $\overline{\beta_{1}}, \beta_{3}, \ldots, \beta_{r}$, i.e. $\left|\beta_{k}\right|<1$ for $k=3,4, \ldots, r$. We denote $\sigma_{k}: \mathbb{Q}\left(\beta_{1}\right) \rightarrow \mathbb{Q}\left(\beta_{k}\right)$ the isomorphism induced by $\beta_{1} \mapsto \beta_{k}$. As $A$ is finite there exists $q \in \mathbb{N}$ such that $q A$ belongs to the ring of integers of the field $\mathbb{Q}(\beta)$. In particular, the norm $N(q a)=q^{r} \prod_{k=1}^{r}\left|\sigma_{k}(a)\right|$ is an integer for any letter $a$ in $A$.

Consider $x, y \in S_{A}(\beta), x \neq y$. Then the difference between $x$ and $y$ can be expressed as $x-y=v=\sum_{j=0}^{n} b_{j} \beta^{j}$, for some $n$ in $\mathbb{N}$ and $b_{j}$ in $A-A$.

Let us denote $A_{k}=\max \left\{\left|\sigma_{k}(a)\right|: a \in A\right\}$. For $k=3,4, \ldots, r$, the modulus of the $k$-th conjugate of $v$ satisfies

$$
\left|\sigma_{k}(v)\right| \leq \sum_{j=0}^{n}\left|b_{j}\right| \cdot\left|\beta_{k}\right|^{j} \leq 2 A_{k} \sum_{j=0}^{\infty}\left|\beta_{k}\right|^{j}=2 A_{k} \frac{\left|\beta_{k}\right|}{1-\left|\beta_{k}\right|} .
$$

Since $\beta$ and $q b_{k}$ are algebraic integers, $q v$ is an algebraic integer as well and its norm is a rational non-zero integer. Compute the norm of $q v$

$$
1 \leq|N(q v)|=q^{r} \prod_{k=1}^{r}\left|\sigma_{k}(v)\right| \leq q^{r} v \bar{v} \prod_{k=3}^{r}\left|\sigma_{k}(v)\right| \leq(2 q)^{r} v \bar{v} \prod_{k=3}^{r} \frac{A_{k}\left|\beta_{k}\right|}{1-\left|\beta_{k}\right|} .
$$

It means that the squared distance $v \bar{v}$ of two different points from the spectrum $S_{A}(\beta)$ is bounded from below by the constant $(2 q)^{-r} \prod_{k=3}^{r} \frac{1-\left|\beta_{k}\right|}{A_{k}\left|\beta_{k}\right|}$. Consequently, the spectrum has no accumulation point.

The case $\beta$ real is analogous.

If the base $\beta$ is real and the alphabet is a symmetric set of consecutive integers, Theorem 3.5 together with the following theorem answers completely the question of the existence of a rigid representation of zero.

Theorem 3.7 (Akiyama and Komornik [1, Feng [9]). Let $\beta>1$ and let $A=$ $\{-M, \ldots, M\}$. Then $S_{A}(\beta)$ has an accumulation point if and only if $\beta<M+1$ and $\beta$ is not Pisot.

If the base $\beta$ is real but the alphabet is not symmetric we have only the following partial observation.

Proposition 3.8. Let $\beta>1$ and $\{-1,0,1\} \subset A=\{m, \ldots, 0, \ldots, M\} \subset \mathbb{Z}$.

1. Zero has a non-trivial $(\beta, A)$-representation if and only if $\beta \leq \max \{M+$ $1,-m+1\}$. 
2. If $\beta \leq \max \{M+1,-m+1\}$, and $\beta$ is not a Parry number, then zero has a rigid $(\beta, A)$-representation.

Proof. Let Let $d_{\beta}(1)=t_{1} t_{2} t_{3} \cdots$ be the greedy expansion of 1 . Then $\beta-1 \leq t_{1}<\beta$, $t_{i} \leq t_{1}$ and

$$
0=0 . \overline{1} t_{1} t_{2} t_{3} \cdots=0.1 \overline{t_{1}} \overline{t_{2}} \overline{t_{3}} \cdots
$$

We have two non-trivial representations of 0 over the alphabets $\{-\lceil\beta\rceil+1, \ldots, \overline{1}, 0,1\}$ and $\{\overline{1}, 0,1, \ldots,\lceil\beta\rceil-1\}$ respectively.

Therefore, if $\left\{-1,0,1, \ldots, t_{1}\right\} \subset A$ or $\left\{-t_{1}, \ldots,-1,0,1,\right\} \subset A$, zero has a nontrivial $(\beta, A)$-representation. Let us note that $t_{1} \in A$ means $M \geq t_{1} \geq \beta-1$. Similarly $-t_{1} \in A$ implies $m \leq-t_{1} \leq-\beta+1$.

On the other hand, let $M<\beta-1$ and $m>-\beta+1$. Then for $z=\sum_{k \geq 1} z_{i} \beta^{-i}$ with $z_{i} \in A$ and $z_{1} \geq 1$, we have $z \geq \frac{1}{\beta}+\sum_{i \geq 2} \frac{m}{\beta}=\frac{\beta-1+m}{\beta(\beta-1)}>0$. Analogously, if $z_{1} \leq-1$, then $z<0$. Consequently, 0 has only the trivial representation.

Now assume that $\beta$ is not a Parry number. Then the sequence of the $n^{\text {th }}$ tails of the $\beta$-expansion of $1, r_{n}=0 . t_{n+1} t_{n+2} \cdots$, is injective. By Lemma 3.4 and Theorem 3.5, zero has a rigid $(\beta, A)$-representation.

Remark 3.9. A numeration system with negative base $-\beta<-1$ and an alphabet $A_{-\beta}=\{0, \ldots,\lfloor\beta\rfloor\}$ was introduced by Ito and Sadahiro in [15. Liao and Steiner in [19] defined an Yrrap number as an analogy of a Parry number for numeration systems with negative base. This definition implies that if $\beta$ is not Yrrap, then there exists a rigid $(-\beta, A)$-representation of 0 over the alphabet $A=\{1, \ldots,\lfloor\beta\rfloor+1\}$.

\section{A problem in automata theory}

\subsection{Representations of 0}

Let $\beta$ be a real number $>1$ We consider infinite $\beta$-representations of 0 on an alphabet of the form $\{-M, \ldots, M\}, M \geq 1$ integer. Let

$$
Z_{\beta, M}=\left\{z_{1} z_{2} \cdots \mid \sum_{i \geq 1} z_{i} \beta^{-i}=0, z_{i} \in\{-M, \ldots, M\}\right\}
$$

be the set of infinite words having value 0 in base $\beta$ on the alphabet $\{-M, \ldots, M\}$.

Proposition 3.8 says that 0 has a non-trivial representation only if $M \geq\lceil\beta\rceil-1$. Therefore, we consider only $M$ satisfying this inequality.

Note that, if $Z_{\beta, M}$ is recognizable by a finite Büchi automaton, then, for every $c<M, Z_{\beta, c}=Z_{\beta, M} \cap\{-c, \ldots, c\}^{\mathbb{N}}$ is recognizable by a finite Büchi automaton as well.

We briefly recall the construction of the (not necessarily finite) Büchi automaton recognizing $Z_{\beta, M}$, see [10] and [12]:

- the set of states is $Q_{M} \subset\left\{\sum_{k=0}^{n} a_{k} \beta^{k} \mid n \in \mathbb{N}, a_{k} \in\{-M, \ldots, M\}\right\} \cap$ $\left[-\frac{M}{\beta-1}, \frac{M}{\beta-1}\right]$

- for $s, t \in Q_{M}, a \in\{-M, \ldots, M\}$ there is an edge

$$
s \stackrel{a}{\longrightarrow} t \Longleftrightarrow t=\beta s+a
$$

- the initial state is 0

- all states are terminal. 


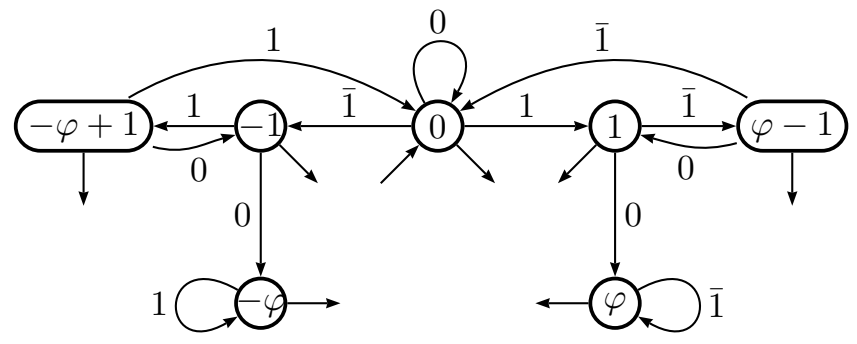

Figure 1: Finite Büchi automaton recognizing $Z_{\varphi, 1}$ for $\varphi=\frac{1+\sqrt{5}}{2}$.

Example 4.1. Take $\beta=\varphi=\frac{1+\sqrt{5}}{2}$ the Golden Ratio. It is a Pisot number, with $d_{\varphi}(1)=11$. A finite Büchi automaton recognizing $Z_{\varphi, 1}$ is designed in Figure1 The initial state is 0 , and all the states are terminal.

Theorem 4.2. Let $\beta>1$ and $A=\{-M, \ldots, M\}$ with $M$ a fixed integer $\geq 1$. The set $Z_{\beta, M}$ is recognizable by a finite Büchi automaton if and only if the spectrum $S_{A}(\beta)$ has no accumulation point.

Proof. To any string $z=z_{1} z_{2} \cdots \in Z_{\beta, M}$ we assigne the sequence of polynomials $P_{n}^{(z)}(X)=z_{1} X^{n-1}+z_{2} X^{n-2}+\cdots+z_{n-1} X+z_{n}$. Denote $R_{n}^{(z)}$ the remainder of the Euclidean division of the polynomial $P_{n}^{(z)}(X)$ by the polynomial $(X-\beta)$. It means that there exists a polynomial $Q_{n}^{(z)}(X)$ such that $P_{n}^{(z)}(X)=(X-\beta) Q_{n}^{(z)}(X)+R_{n}^{(z)}$. Clearly $P_{n}^{(z)}(\beta)=R_{n}^{(z)}$. Denote $R=\left\{R_{n}^{(z)}: z \in Z_{\beta, M}\right.$ and $\left.n \in \mathbb{N}\right\}$.

As $z=z_{1} z_{2} \cdots$ is a $(\beta, A)$-representation of 0 , the value $P_{n}^{(z)}(\beta)=-0 \cdot z_{n+1} z_{n+2} \cdots$ belongs to the spectrum $S_{A}(\beta)$ and $-P_{n}^{(z)}(\beta)$ is the $n^{\text {th }}$ tail $r_{n}$ of the $(\beta, A)$ representation of 0 . Consequently,

$$
R \subset S_{A}(\beta) \text { and } \quad R \text { is bounded. }
$$

To prove the theorem, we apply Proposition 3.1 from [10. It says that $Z_{\beta, M}$ is recognizable by a finite Büchi automaton if and only if the set $R$ is finite.

$(\Leftarrow)$ If $Z_{\beta, M}$ is not recognizable by finite automaton, then $R$ is infinite and by (4.1) the spectrum has an accumulation point.

$(\Rightarrow)$ If $S_{A}(\beta)$ has an accumulation point, then by Theorem 3.5, zero has a rigid representation $z_{1} z_{2} \cdots \in Z_{\beta, M}$. By Point 2 of Lemma 3.4 the sequence of its tails $\left(r_{n}\right)$ is injective. Since $-r_{n}=P_{n}^{(z)}(\beta)=R_{n}^{(z)} \in R$, the set $R$ is not finite and therefore $Z_{\beta, M}$ is not recognizable by finite automaton.

Combining Theorems 3.7 and 4.2, we answer a conjecture raised in [12 and obtain the following result.

Theorem 4.3. Let $\beta>1$. The following conditions are equivalent:

1. the set $Z_{\beta, M}$ is recognizable by a finite Büchi automaton for every positive integer $M$,

2. the set $Z_{\beta, M}$ is recognizable by a finite Büchi automaton for one $M \geq\lceil\beta\rceil-1$,

3. $\beta$ is a Pisot number.

Remark 4.4. The fact that, if $\beta$ is not a Pisot number, then the set $Z_{\beta, M}$ is not recognizable by a finite Büchi automaton for any $M \geq\lceil\beta\rceil$ was already settled in Theorem 1.2, but the proof given above is more direct than the original one. 


\subsection{Normalization}

Normalization in base $\beta$ is the function which maps a $\beta$-representation on the canonical alphabet $A_{\beta}=\{0, \ldots,\lceil\beta\rceil-1\}$ of a number $x \in[0,1]$ onto the greedy $\beta$ expansion of $x$. From the Büchi automaton $\mathcal{Z}$ recognizing the set of representations of 0 on the alphabet $\{-\lceil\beta\rceil+1, \ldots,\lceil\beta\rceil-1\}$, one constructs a Büchi automaton (a converter) $\mathcal{C}$ on the alphabet $A_{\beta} \times A_{\beta}$ that recognizes the set of couples on $A_{\beta}$ that have the same value in base $\beta$, as follows:

$$
s \stackrel{(a, b)}{\longrightarrow} t \text { in } \mathcal{C} \Longleftrightarrow s \stackrel{a-b}{\longrightarrow} t \text { in } \mathcal{Z},
$$

see [12] for details. Obviously $\mathcal{C}$ is finite if and only if $\mathcal{Z}$ is finite.

Then we take the intersection of the set of second components with the set of greedy $\beta$-expansions of the elements of $[0,1]$, which is recognizable by a finite Büchi automaton when $\beta$ is a Pisot number, see [4]. Thus the following result holds true.

Corollary 4.5. Normalization in base $\beta>1$ is computable by a finite Büchi automaton on the alphabet $A_{\beta} \times A_{\beta}$ if and only if $\beta$ is a Pisot number.

\section{On-line division in complex base}

\subsection{Trivedi-Ercegovac algorithm}

On-line arithmetic, introduced in [25], is a mode of computation where operands and results are processed in a digit serial manner, starting with the most significant digit. To generate the first digit of the result, the first $\delta$ digits of the operands are required. The integer $\delta$ is called the delay of the algorithm.

In [5, 6] we have extended the original on-line algorithm of Trivedi-Ercegovac to the complex case.

The algorithm for on-line division in a complex numeration system $(\beta, A)$ has two parameters: the delay $\delta \in \mathbb{N}$ and $D>0$, the minimal value (in modulus) of the divisor.

The $(\beta, A)$-representation of the nominator is $n=\sum_{i=1}^{\infty} n_{i} \beta^{-i}$, of the divisor is $d=\sum_{i=1}^{\infty} d_{i} \beta^{-i}$, and of their quotient $q=\sum_{i=1}^{\infty} q_{i} \beta^{-i}$. Partial sums are denoted by $N_{k}=\sum_{i=1}^{k} n_{i} \beta^{-i}, D_{k}=\sum_{i=1}^{k} d_{i} \beta^{-i}$, and $Q_{k}=\sum_{i=1}^{k} q_{i} \beta^{-i}$.

The inputs of the algorithm are two infinite strings $0 . n_{1} n_{2} \cdots n_{\delta} n_{\delta+1} n_{\delta+2} \cdots$ with $n_{i} \in A$ and $n_{1}=n_{2}=\cdots=n_{\delta}=0$ and $0 . d_{1} d_{2} \cdots$ with $d_{i} \in A$ satisfying $\left|D_{j}\right| \geq D$ for all $j \in \mathbb{N}, j \geq 1$.

The output is a string $q_{1} q_{2} q_{3} \ldots$ corresponding to a $(\beta, A)$-representation of the quotient $q=n / d=0 \cdot q_{1} q_{2} q_{3} \cdots$. The settings of the algorithm ensure that the representation of $q$ starts behind the fractional point.

Set $W_{0}=q_{0}=Q_{0}=0$. Then, for $k \geq 1$ compute

$$
W_{k}=\beta\left(W_{k-1}-q_{k-1} D_{k-1+\delta}\right)+\left(n_{k+\delta}-Q_{k-1} d_{k+\delta}\right) \beta^{-\delta} .
$$

The $k$-th digit $q_{k}$ of the representation of the quotient is evaluated by a function Select, function of the values of the auxiliary variable $W_{k}$ and the interim representation $D_{k+\delta}$, so that

$$
q_{k}=\operatorname{Select}\left(W_{k}, D_{k+\delta}\right) \in A .
$$

It can be shown that for any $k \geq 1$ :

$$
W_{k}=\beta^{k}\left(N_{k+\delta}-Q_{k-1} D_{k+\delta}\right) .
$$

Moreover, if the sequence $\left(W_{k}\right)$ is bounded, then $q=\lim _{k \rightarrow \infty} Q_{k}=\frac{n}{d}$.

Conditions on the system $(\beta, A)$ so that the definition of the function Select ensures the correctness of the on-line division algorithm are given in [5, 6]. 


\subsection{Preprocessing of divisors}

When making division, we need that the divisor stays away from 0 . By definition of the on-line algorithm, this means that the value of all the prefixes of the divisor $d_{1} d_{2} \cdots$ must be greater in absolute value than a parameter $D>0$.

Definition 5.1. We say that a complex numeration system $(\beta, A)$ allows preprocessing if there exists $D>0$ and a finite list $\mathcal{L}$ of identities of the type $0 \cdot w_{k} \cdots w_{0}=$ $0.0 u_{k-1} \cdots u_{0}$ with digits in $A$ such that any string $d_{1} d_{2} \cdots$ on $A$ without prefix $w_{k} \cdots w_{0}$ from $\mathcal{L}$ satisfies $\left|0 . d_{1} d_{2} \cdots d_{j}\right|>D$ for all $j \in \mathbb{N}$.

We must have at least $d_{1} \neq 0$ after preprocessing, so the preprocessing consists first of all in shifting the fractional point to the most significant non-zero digit of the $(\beta, A)$-representation of the divisor. Of course, after preprocessing the value of the original divisor $w$ has been changed into a new one $d$ which is just a shift of the original one, that is to say $d=w \beta^{k}$ for some $k$ in $\mathbb{Z}$. This will have to be taken into account to give the result of the division.

If zero has only the trivial $(\beta, A)$-representation the situation is simple. This fact can be equivalently rewritten as

$$
\inf \mathcal{R}>0, \text { where } \mathcal{R}=\left\{\left|\sum_{i \geq 1} z_{i} \beta^{-i}\right|: z_{1} \neq 0, z_{i} \in A\right\} .
$$

In this case the numeration system $(\beta, A)$ allows preprocessing, since we can take $D=\inf \mathcal{R}$ and the list of rewriting rules is empty.

Example 5.2. If $\beta=4$ and $A=\{\overline{2}, \overline{1}, 0,1,2\}$, then zero has only the trivial representation and for $D$ one can take $\frac{1}{12}=\min \mathcal{R}$.

Example 5.3. If $\beta=2$ and $A=\{\overline{1}, 0,1\}$, zero has two non-trivial representations $0=0.1 \overline{1} \overline{1} \overline{1} \cdots=0 . \overline{1} 1111 \cdots$. Therefore, preprocessing is a little bit more sophisticated. Consider the list

$$
0 . \overline{1} 1=0.0 \overline{1} \text { and } 0.1 \overline{1}=0.01
$$

If a string $d_{1} d_{2} \cdots$ has no prefix $\overline{1} 1$ neither $1 \overline{1}$, then

$$
\left|0 . d_{1} d_{2} \cdots d_{j}\right| \geq 0.10 \overline{1} \overline{1} \overline{1} \cdots=\frac{1}{4}
$$

and thus one can take $D=\frac{1}{4}$.

Theorem 5.4. A complex numeration system $(\beta, A)$ allows preprocessing if and only if the spectrum $S_{A}(\beta)$ has no accumulation point.

The result is proved by the following three lemmas, in which we use the notation

$$
H=\max \left\{\left|\sum_{i \geq 1} d_{i} \beta^{-i}\right|: d_{i} \in A \text { for all } i \in \mathbb{N}\right\} .
$$

Lemma 5.5. If 0 has a rigid $(\beta, \mathcal{A})$-representation then the numeration system $(\beta, \mathcal{A})$ does not allow preprocessing.

Proof. Let $0=0 \cdot z_{1} z_{2} z_{3} \cdots$ be a rigid representation of 0 . Assume that preprocessing is possible with $D>0$. Find $j$ such that $\frac{H}{|\beta|^{j}}<D$. Consider the number $0 . z_{1} z_{2} z_{3} \cdots z_{j} 000 \cdots$. Since the representation of zero is rigid, no prefix of the string $z_{1} z_{2} z_{3} \cdots z_{j}$ is contained in the list of the rewriting rules. But $\left|0 . z_{1} z_{2} z_{3} \cdots z_{j}\right|=|0 \cdot \underbrace{00 \cdots 0}_{j \text {-times }} z_{j+1} z_{j+2} \cdots|<\frac{H}{|\beta|^{j}}<D$ - a contradiction. 
Lemma 5.6. Let us assume that $S_{A}(\beta)$ has no accumulation point and fix $K>0$. Then there exists $m \in \mathbb{N}$ such that any string $x_{m-1} x_{m-2} \cdots x_{1} x_{0}$ of length $m$ over A satisfies either

$$
\left|x_{m-1} \beta^{m-1}+x_{m-2} \beta^{m-2}+\cdots+x_{1} \beta+x_{0}\right| \geq K
$$

or there exists a string $y_{k-1} x_{k-2} \cdots y_{1} y_{0}$ of length $k<m$ over $A$ such that

$x_{m-1} \beta^{m-1}+x_{m-2} \beta^{m-2}+\cdots+x_{1} \beta+x_{0}=y_{k-1} \beta^{k-1}+y_{k-2} \beta^{k-2}+\cdots+y_{1} \beta+y_{0}$.

Proof. Since $S_{A}(\beta)$ has no accumulation point, the set $P=\left\{z \in S_{A}(\beta):|z|<K\right\}$ is finite. Denote $m=1+\max \{\rho(z): z \in P\}$. Let $x=x_{m-1} \beta^{m-1}+x_{m-2} \beta^{m-2}+$ $\cdots+x_{1} \beta+x_{0}$. Obviously, $x \in S_{A}(\beta)$. Then either $|x| \geq K$ or $x \in P$ and thus $x=y_{k} \beta^{k-1}+y_{k-2} \beta^{k-2}+\cdots+y_{1} \beta+y_{0}$, where $k \leq \max \{\rho(z): z \in P\} \leq m-1$.

Lemma 5.7. If $S_{A}(\beta)$ has no accumulation point, then there exists $D>0$ and $m \in \mathbb{N}$ such that for all infinite strings $d_{1} d_{2} \cdots$ over $A$ one has

1. either $\left|0 . d_{1} d_{2} \cdots d_{j}\right| \geq D$ for all $j \in \mathbb{N}$,

2. or $0 . d_{1} d_{2} \cdots d_{m}=0.0 d_{2}^{\prime} d_{3}^{\prime} \cdots d_{m}^{\prime}$ for some string $d_{2}^{\prime} d_{3}^{\prime} \cdots d_{m}^{\prime} \in A^{*}$.

Proof. Let us take $\mu>0$ and apply Lemma [5.6 with $K=H+\mu$ to get $m \in \mathbb{N}$. Denote $\mathcal{D}=\left\{\left|0 . d_{1} d_{2} \cdots d_{j}\right|: j<m\right.$ and $\left.\quad 0 . d_{1} d_{2} \cdots d_{j} \neq 0.0 d_{2}^{\prime} \cdots d_{j}^{\prime}\right\}$. The set $\mathcal{D}$ is finite and does not contain zero. Therefore, $D^{\prime}=\min \mathcal{D}>0$.

To prove the lemma, consider an infinite string $d_{1} d_{2} \cdots$ and assume that $0 . d_{1} d_{2} \cdots d_{m} \neq$ $0.0 d_{2}^{\prime} d_{3}^{\prime} \cdots d_{m}^{\prime}$ for all strings $d_{2}^{\prime} d_{3}^{\prime} \cdots d_{m}^{\prime} \in A^{*}$. We distinguish two cases

- $j<m, j \in \mathbb{N}$. Then $0 . d_{1} d_{2} \cdots d_{j} \neq 0.0 d_{2}^{\prime} \cdots d_{j}^{\prime}$, otherwise $0 . d_{1} d_{2} \cdots d_{m}=$ $0.0 d_{2}^{\prime} d_{3}^{\prime} \cdots d_{j}^{\prime} d_{j+1} \cdots d_{m}-$ a contradiction. Therefore, $\left|0 . d_{1} d_{2} \cdots d_{j}\right| \geq D^{\prime}$.

- $j \geq m, j \in \mathbb{N}$. Then

$$
\left|0 \cdot d_{1} d_{2} \cdots d_{j}\right| \geq\left|0 \cdot d_{1} d_{2} \cdots d_{m}\right|-\frac{1}{|\beta|^{m}}\left|0 \cdot d_{m+1} d_{m+2} \cdots d_{j}\right| \geq \frac{1}{|\beta|^{m}} K-\frac{1}{|\beta|^{m}} H=\frac{\mu}{|\beta|^{m}}
$$

Thus we can set $D=\min \left\{D^{\prime}, \frac{\mu}{|\beta|^{m}}\right\}$.

The previous lemma gives a hint for creating the list of rewriting rules. We take the index $m$ found by the lemma and inspect all strings $d_{1} d_{2} \cdots d_{m}$ over $A$. If $0 . d_{1} d_{2} \cdots d_{m}=0.0 d_{2}^{\prime} d_{3}^{\prime} \cdots d_{m}^{\prime}$ for some string $d_{2}^{\prime} d_{3}^{\prime} \cdots d_{m}^{\prime}$ we put it into the list.

Example 5.8. Let $\beta=\varphi=\frac{1+\sqrt{5}}{2}$ and $A=\{\overline{1}, 0,1\}$. The minimal polynomial of $\varphi$ is $X^{2}-X-1$. In this numeration system, 0 has countably many finite representations and uncountably many infinite representations. As the alphabet is symmetric, the rewriting rules appear in pairs. For example, as $10 \overline{1}$ can be rewritten to 010, also $\overline{1} 01$ can be rewritten to $0 \overline{1} 0$. To shorten our list, we put into it only one rule of each pair, namely the rule, where the first digit is 1 . First we consider the list

$\mathcal{L}_{0}: 10 \overline{1} \longrightarrow 010,1 \overline{1} 0 \longrightarrow 001,1 \overline{11} \longrightarrow 000$.

Claim: If no rule from $\mathcal{L}_{0}$ can be applied to the string $d_{1} d_{2} \cdots$, then $|d| \geq D=\frac{1}{\varphi^{5}}$, where $d=0 . d_{1} d_{2} \cdots$.

Proof. WLOG $d_{1}=1$.

If $d_{2}=0$, then $\geq 0$ and thus $|d| \geq \frac{1}{\varphi}-\sum_{k \geq 4}^{\infty} \varphi^{-k}=\frac{1}{\varphi}-\frac{1}{\varphi^{2}}=\frac{1}{\varphi^{3}} \geq D$.

If $d_{2}=1$, then $|d| \geq \frac{1}{\varphi}+\frac{1}{\varphi^{2}}-\sum_{k \geq 3}^{\infty} \varphi^{-k}=1-\frac{1}{\varphi}-=\frac{1}{\varphi^{2}} \geq D$.

If $d_{2}=\overline{1}$, then $d_{3}=1$. Therefore, $|d| \geq \frac{1}{\varphi}-\frac{1}{\varphi^{2}}+\frac{1}{\varphi^{3}}-\sum_{k \geq 4}^{\infty} \varphi^{-k}=\frac{1}{\varphi^{5}} \geq D$. 
We can extend the list of rewriting rules to increase the lower bound $D$. Let us consider the whole families of rules $\mathcal{L}$ :

$(1 \overline{1})^{k} 0 \longrightarrow 00(10)^{k-1} 1 \quad$ for $k \geq 1$.

$(1 \overline{1})^{k} \overline{1} \longrightarrow 00(10)^{k-1} 0 \quad$ for $k \geq 1$.

$(1 \overline{1})^{k} 10 \overline{1} \longrightarrow 01(00)^{k} 0 \quad$ for $k \geq 0$.

$(1 \overline{1})^{k} 100 \longrightarrow 01(00)^{k} 1 \quad$ for $k \geq 0$.

$(1 \overline{1})^{k} 11 \longrightarrow 01(00)^{k-1} 10 \quad$ for $k \geq 1$.

$10 \overline{1}^{k} 0 \longrightarrow 0^{k+1} 11 \quad$ for $k \geq 0$.

$10 \overline{1}^{k} 1 \longrightarrow 0^{k} 101 \quad$ for $k \geq 1$.

Claim: If no rule from $\mathcal{L}$ can be applied to the string $d_{1} d_{2} \cdots$, then $|d| \geq D=\frac{1}{\varphi^{2}}$, where $d=0 . d_{1} d_{2} \cdots$.

Proof. WLOG $d_{1}=1$. Our string has a prefix 11 or a prefix $(1 \overline{1})^{k} 101$ for $k \geq 0$. Therefore either

$$
|d| \geq 0.11(\overline{1})^{\omega}=\frac{1}{\varphi^{2}} \quad \text { or } \quad|d| \geq 0 \cdot(1 \overline{1})^{k} 101(\overline{1})^{\omega}=\frac{1}{\varphi^{2}}+\frac{1}{\varphi^{2 k+3}} .
$$

Some examples where the base is a complex number can be found in [6].

\section{Comments and open questions}

\subsection{F-number}

In [18] Lau defined for $1<\beta<2$ the following notion, that we extend to any $\beta>1$.

Definition 6.1. Let $\beta>1$ and $B_{\beta}=\{-\lceil\beta\rceil+1, \ldots,\lceil\beta\rceil-1\}$ be the symmetrized alphabet of the canonical alphabet $A_{\beta}$. Then $\beta$ is said to be a $F$-number if the set

$$
L_{(\lceil\beta\rceil-1)}(\beta)=S_{B_{\beta}}(\beta) \cap\left[-\frac{\lceil\beta\rceil-1}{\beta-1}, \frac{\lceil\beta\rceil-1}{\beta-1}\right]
$$

is finite.

Feng proved in 9] that $1<\beta<2$ is a F-number if and only if it is a Pisot number. This property extends readily to any $\beta>1$. Another way of proving it consists in realizing that the set of states $Q_{(\lceil\beta\rceil-1)}$ of the automaton for $Z_{\beta,\lceil\beta\rceil-1}$ is included into $L_{(\lceil\beta\rceil-1)}(\beta)$.

\subsection{Open questions}

- A motivation for introducing the notion of "rigid representation of zero" comes from on-line division in a numeration system $(\beta, A)$. A more elementary question is "Has zero a non-trivial $(\beta, A)$-representation"? The answer is easy for real bases and alphabets of the form $A=\{m, \ldots, 0, \ldots, M\}$, see Proposition 3.8. The same question for complex bases is an open problem.

- In the case that the base is real and the alphabet is $A=\{-M, \ldots, M\}$, Theorem 4.2 says that recognizability by a finite automaton is equivalent to the fact that the spectrum $S_{A}(\beta)$ has no accumulation point.

An analogous result can be proved for complex bases as well. But for complex bases the question about the existence of accumulation points in the spectrum $S_{A}(\beta)$ is not yet investigated. Nevertheless, it is often easy to check that a $(\beta, A)$-representation of 0 is not rigid. 
- If $\beta>1$ is a non-Pisot base then $A=\{-\lceil\beta\rceil+1, \ldots,\lceil\beta\rceil-1\}$ is the smallest symmetric alphabet of consecutive integers for which the spectrum $S_{A}(\beta)$ has an accumulation point. What is the minimal size of an alphabet $A=$ $\{-M, \ldots, M\} \subset \mathbb{Z}$ for which the spectrum of a non-Pisot complex number $\beta$ has an accumulation point?

\section{Acknowledgements}

Ch. F. acknowledges support of ANR/FWF project "FAN", grant ANR-12-IS010002 .

\section{References}

[1] S. Akiyama and V. Komornik, Discrete spectra and Pisot numbers, J. Number Theory 133 (2013) 375-390.

[2] D. Berend and Ch. Frougny, Computability by finite automata and Pisot bases, Math. Systems Theory 27 (1994) 274-282.

[3] A. Bertrand, Développements en base de Pisot et répartition modulo 1, C. R. Acad. Sci. Paris, Sér. A 285 (1977) 419-421.

[4] A. Bertrand-Mathis, Développements en base $\theta$, répartition modulo un de la suite $\left(x \theta^{n}\right)_{n \geq 0}$, langages codés et $\theta$-shift, Bull. Soc. Math. Fr. 114 (1986) 271323.

[5] M. Brzicová, Ch. Frougny, E. Pelantová, and M. Svobodová, On-line multiplication and division in real and complex bases, in Proceedings of IEEE ARITH 23, I.E.E.E. Computer Society Press (2016) 134-141.

[6] M. Brzicová, Ch. Frougny, E. Pelantová, and M. Svobodová, On-line algorithms for multiplication and division in real and complex numeration systems, arXiv:1610.08309, Oct. 2016.

[7] Y. Bugeaud, On a property of Pisot numbers and related questions, Acta Math. Hung. 73 (1996) 33-39.

[8] P. Erdös, I. Joó and V. Komornik, On the sequence of numbers of the form $\epsilon_{0}+\epsilon_{1} q+\cdots+\epsilon_{n} q^{n}, \epsilon_{i} \in\{0,1\}$, Acta Arith. 83 (1998) 201-210.

[9] D.-J. Feng, On the topology of polynomials with bounded integer coefficients, J. Eur. Math. Soc. 18 (2016) 181-193.

[10] Ch. Frougny, Representation of numbers and finite automata, Math. Systems Theory 25 (1992) 37-60.

[11] Ch. Frougny and J. Sakarovitch, Automatic conversion from Fibonacci representation to representation in base $\varphi$, and a generalization, Internat. J. Algebra Comput. 9 (1999) 351-384.

[12] Ch. Frougny and J. Sakarovitch, Number representation and finite automata, Chapter 2 in Combinatorics, Automata and Number Theory, C.U.P., 2010.

[13] A. M. Garsia, Arithmetic properties of Bernoulli convolutions, Trans. Amer. Math. Soc. 102 (1962) 409-432. 
[14] W. J. Gilbert, Radix representations of quadratic fields, J. Math. Anal. Appl. 83 (1981) 264-274.

[15] S. Ito and T. Sadahiro, Beta-expansions with negative bases, Integers 9 (2009), A22, 239-259.

[16] I. Kátai and B. Kovács, Canonical number systems in imaginary quadratic fields, Acta Math. Acad. Sci. Hungar. 37 (1981) 159-164.

[17] D. E. Knuth, An imaginary number system, Communications of the ACM 3 (1960) 245-247.

[18] K.-S. Lau, Dimension of a family of singular Bernoulli convolutions, J. Funct. Anal. 116 (1993) 335-358.

[19] L. Liao and W. Steiner, Dynamical properties of the negative betatransformation, Ergod. Theory Dyn. Syst 32(5) (2012) 1673-1690.

[20] W. Parry, On the $\beta$-expansions of real numbers, Acta Math. Acad. Sci. Hungar. 11 (1960) 401-416.

[21] A. Rényi, Representations for real numbers and their ergodic properties, Acta Math. Acad. Sci. Hungar. 8 (1957) 477-493.

[22] M. Rigo, Formal languages, automata and numeration systems, volume 1: Introduction to combinatorics on words. ISTE-Wiley, 2014.

[23] K. Schmidt, On periodic expansions of Pisot numbers and Salem numbers, Bull. London Math. Soc. 12 (1980) 269-278.

[24] W. P. Thurston, Groups, tilings and finite automata, Geometry Supercomputer Project Research Report GCG1, University of Minnesota, 1989.

[25] K. S. Trivedi and M. D. Ercegovac, On-Line Algorithms for Division and Multiplication, IEEE Transactions on Computers C-26 (1977) 681-687. 